\title{
Compromiso nodal y extranodal detectado con PET/CT en linfoma en etapificación
}

\author{
David Ladrón de Guevara $H^{1}$, Gabriel Briceño ${ }^{2}$, Sebastián Cid $H^{2}$, Felipe Durán $V^{2}$, Laura ltriago $G^{3}$, \\ Manuel Álvarez Z3.
}

1. Departamento de Radiología, Clínica Las Condes. Santiago, Chile.

2. Alumno de Medicina. Facultad de Medicina, Universidad de Chile. Santiago, Chile.

3. Centro Clínico de Cáncer, Clínica Las Condes. Santiago, Chile.

\section{Nodal and extranodal involvement detected by PET/CT in staging lymphoma}

\begin{abstract}
Objective: To describe visible nodal and extra-nodal involvement using PET/CT in the different types and subtypes of lymphoma in staging. Patients and method: PET/CT with F18-FDG were reviewed in patients with lymphoma staging, determining frequency and location of nodal and extra-nodal involvement, and intensity of F18-FDG uptake measured by SUVmax. Results: Of the 102 patients with NHL (average SUVmax $13.0 \pm 9.7$ ), $86.3 \%$ had nodal involvement (51.9\% on both sides of the diaphragm, $24.5 \%$ only above the diaphragm), and $66.7 \%$ extra-nodal compromise (42.6\% bone marrow, $22.1 \%$ muscle, $16.2 \%$ renal). Of the 30 patients with HL (average SUVmax $14.6 \pm 6.0), 100 \%$ had nodal involvement (63.3\% only above the diaphragm, $36.7 \%$ above and below the diaphragm), and $30 \%$ had extra-nodal involvement (66.7\% bone marrow, $22.2 \%$ lung). Conclusion: PET/CT is the method of choice in the staging of lymphoma, allowing the detection of nodal and extra-nodal disease in both $H L$ and NHL.
\end{abstract}

Keywords: Lymphoma, Hodgkin, non-Hodgkin, PET/CT, nodal, extranodal.

Resumen. Objetivo: Describir el compromiso nodal y extranodal visible con PET/CT en los distintos tipos y subtipos de linfoma en etapificación. Pacientes y método: Se revisaron los PET/CT con F18-FDG realizados a pacientes con linfoma en etapificación, determinando frecuencia y localización del compromiso nodal y extranodal, e intensidad de captación de F18-FDG medida mediante SUVmax. Resultados: De los 102 pacientes con $L N H$ (SUVmax promedio $13,0 \pm 9,7)$, un $86,3 \%$ presentó compromiso nodal (51,9\% a ambos lados del diafragma, $24,5 \%$ sólo sobre el diafragma) y 66,7\% compromiso extranodal (42,6\% médula ósea, $22,1 \%$ muscular, 16,2\% renal). De los 30 pacientes con LH (SUVmax promedio 14,6 66,0 ), el 100\% tuvo compromiso nodal (63,3\% sólo sobre el diafragma, 36,7\% sobre y bajo el diafragma), y 30\% compromiso extranodal (66,7\% médula ósea, 22,2\% pulmón). Conclusión: El PET/CT es el método de elección en la etapificación del linfoma, permitiendo detectar enfermedad nodal y extranodal, tanto en LH como en LNH. Palabras clave: Linfoma, Hodgkin, No Hodgkin, PET/CT, nodal, extranodal.

Ladrón de Guevara D., et al. Compromiso nodal y extranodal detectado con PET/CT en linfoma en etapificación. Rev Chil Radiol 2017; 23(3): 91-97.

Correspondencia: David Ladrón de Guevara Hernández/dlg@clc.cl

Trabajo enviado 16 de agosto de 2017. Aceptado para publicación el 16 de septiembre de 2017.

\section{Introducción}

El término linfoma se utiliza para identificar un grupo heterogéneo de neoplasias que se originan en las células de los órganos del tejido linfoide y que tienen comportamiento biológico y clínico diferente. Históricamente divididas en 2 grandes categorías:
Linfoma de Hodgkin (LH) y Linfoma No Hodgkin $(\mathrm{LNH})^{1}$. Aun cuando ambas neoplasias son sensibles a la quimioterapia y a la radioterapia, sus tasas de curación son diferentes. EI LH puede curarse hasta en un $80 \%$ de los pacientes empleando tratamientos tradicionales y terapias de rescate, el LNH se cura en 
menos del $50 \%^{2}$. Los linfomas se encuentran dentro de los cánceres más frecuentes en pacientes con edades comprendidas entre los 20 y 40 años.

El LH representa el $10 \%$ de todos los linfomas, tiene una curva de edad de distribución bimodal, con un peak de incidencia entre los 20 y 30 años y otro a los 65 años. Leve predominancia en el sexo masculino. El LH se caracteriza por la presencia de células de Reed Sternberg, que provienen de células $B$ del centro germinal, rodeadas de un infiltrado celular reactivo.

La mayoría de los pacientes con LH se presentan con un aumento de volumen o masa ganglionar asintomática, que se disemina de forma ordenada hacia áreas ganglionares continuas, con baja frecuencia de compromiso extranodal, el que ocurre tarde en el curso de la enfermedad ${ }^{3}$.

EI LNH consiste en un grupo de enfermedades que se produce por la transformación neoplásica de células B maduras, células T y Natural Killer. Se ha propuesto una célula de origen para cada tipo de LNH de acuerdo a su morfología, inmunofenotipo y citogenética. Los subtipos más frecuentes de LNH son: Linfoma difuso de células grandes, Linfoma del manto, Linfoma de Burkitt, Linfoma linfocítico/ leucemia linfática crónica (LLC), Linfoma folicular, Linfoma MALT, Linfoma linfoplasmocitario, Linfoma zona marginal, Leucemia/linfoma T del Adulto.

El LNH es 6 veces más frecuente que el LH, predomina en el sexo masculino y en caucásicos, su incidencia aumenta con la edad, especialmente después de los 40 años. La presentación clínica varía de acuerdo al subtipo de linfoma y a las áreas involucradas. Según series basadas en imágenes convencionales, el 10 a $35 \%$ tiene compromiso extranodal al momento del diagnóstico ${ }^{4}$.

El estudio inicial de los pacientes con Linfoma se basa fuertemente en exámenes de imagen, los que deben ser capaces de determinar la localización, extensión y tamaño de las lesiones. La tomografía computada (TC) ha sido empleada con relativo éxito en la etapificación de pacientes con linfoma, dada su amplia disponibilidad y excelente calidad de imagen, otorgando una óptima evaluación estructural y anatómica. Sin embargo, desde la aparición del PET (Positron Emission Tomography) y especialmente del PET/CT (PET/Computed Tomography), numerosos estudios comparativos han establecido su mayor rendimiento diagnóstico respecto a la $\mathrm{TC}^{5-7}$.

EI PET utiliza como radiotrazador la F18-Fluorodeoxiglucosa (F18-FDG), que es una molécula de glucosa marcada con un isótopo de Flúor que emite radiación, y que permite visualizar su distribución en el organismo. Cada tipo de linfoma tiene una avidez de F18-FDG diferente, siendo los más captantes los $\mathrm{LH}$ y aquellos $\mathrm{LNH}$ de comportamiento agresivo ${ }^{7,8}$. La sensibilidad del PET/CT en la detección de lesiones dependerá del grado de captación de glucosa marcada que presenta el linfoma en estudio.

Schaefer y cols ${ }^{5}$ demostraron una mayor sensibilidad y especificidad del PET/CT (94\% y 100\%) versus la TC contrastada ( $88 \%$ y $86 \%$ ) en la evaluación del compromiso ganglionar en LH y LNH difuso de células grandes, que son dos de los tipos de linfoma más ávidos de F18-FDG. Ellos también obtuvieron una diferencia aun mayor al evaluar el compromiso extraganglionar, con una sensibilidad y especificidad del PET/CT de $88 \%$ y $100 \%$, respectivamente, versus $50 \%$ y $90 \%$ de la TC.

La capacidad de detectar más lesiones nodales y extranodales que las técnicas tradicionales, y su consecuente impacto en la etapificación inicial, y por lo tanto en el pronóstico y manejo de estos pacientes, ha hecho que el PET/CT sea considerado actualmente el examen de elección en la evaluación inicial de la $\mathrm{LH}$ y de algunos de los $\mathrm{LNH}^{7,8}$.

El objetivo de este estudio es describir los hallazgos del PET/CT en una población con linfoma recientemente diagnosticado, particularmente el compromiso nodal/extranodal y los distintos grados de avidez de F18-FDG de cada tipo de linfoma.

\section{Pacientes y método}

Se revisaron los exámenes PET/CT realizados en Clínica Las Condes entre diciembre de 2008 y diciembre de 2015, seleccionando aquellos solicitados a pacientes con linfoma en etapificación, que no hubieran recibido quimioterapia o radioterapia previas. Se rescató el estudio histológico de los pacientes, determinando tipo y subtipo histológico en cada caso. Los casos de histología compleja fueron consensuados entre dos patólogos expertos, y paralelamente enviados a un centro de referencia para su análisis (CDM Fletcher, M.D., Brigham and Women's Hospital). Los estudios seleccionados se clasificaron basados en los criterios $\mathrm{WHO}^{9}$.

\section{Aspectos técnicos del PET/CT}

El examen PET/CT se realizó en equipo GE Discovery STE 16 canales, 1 hora después de administrar F18-FDG en dosis de $0,1 \mathrm{mCi} / \mathrm{Kg}$ por vía endovenosa. Inmediatamente después del PET/CT, se realizó en el mismo equipo una tomografía computada contrastada de cerebro, cuello, tórax, abdomen y pelvis en todos los casos, previa administración de medio de contraste iodado endovenoso.

\section{Análisis de las imágenes}

Las imágenes de PET/CT fueron examinadas por un doble especialista radiólogo/médico nuclear, registrando: Ubicación, tamaño e intensidad de captación de F18-FDG de cada lesión. El grado de captación de F18-FDG fue estimado utilizando el índice SUVmax (Standarized Uptake Value maximum) ${ }^{10}$. 


\section{Extensión del linfoma}

Para determinar extensión de la enfermedad se utilizaron criterios de Ann Arbor de acuerdo a las nuevas recomendaciones ${ }^{6}$. Se consideró enfermedad nodal aquella que afecta linfonodos o tejido linfoide (amígdalas, anillo de Waldeyer, bazo, timo), y enfermedad extranodal a cualquier otro compromiso fuera de estas localizaciones ${ }^{6,11}$. Se consideró compromiso esplénico a la hipercaptación difusa (mayor al hígado), focal/multifocal, o ambas.

Se definió Bulky como una masa $\geq 10 \mathrm{~cm}$ o lesión mediastinica mayor a $33 \%$ del diámetro transverso interno del tórax medido a nivel T5-T6 ${ }^{12}$.

De acuerdo a los criterios WHO, se definió LNH subtipo Doble Hit al linfoma difuso células grandes (DCG) tipo B con caracteres intermedios entre DCG y Burkitt ${ }^{9}$.

\section{Estudio estadístico}

Al tratarse de un estudio descriptivo, se requirió solo análisis estadístico simple utilizando t-test, Mann Withney y comparación de proporciones según correspondiera. Para estudio de correlación se empleó Spearman Rank Order.

\section{Aspectos éticos}

El estudio contó con la aprobación del Comité de Ética de la Institución, con acta emitida el 25 de

Tabla 1. Frecuencia de compromiso extranodal según tipo y subtipo histológico de linfoma.

\begin{tabular}{|c|c|c|c|}
\hline & & \multicolumn{2}{|c|}{ Compromiso EN } \\
\hline & & $\mathrm{n}$ & $\%$ \\
\hline LNH & $\mathrm{n}$ & & \\
\hline Difuso Cels Grandes & 34 & 26 & 76,5 \\
\hline Burkitt & 2 & 2 & 100 \\
\hline Doble Hit & 4 & 3 & 75 \\
\hline Folicular & 16 & 7 & 43,8 \\
\hline Linfocítico & 8 & 1 & 12,5 \\
\hline MALT & 8 & 8 & 100 \\
\hline Manto & 8 & 2 & 25 \\
\hline Transformación & 4 & 4 & 100 \\
\hline Otros LNH B & 9 & 8 & 88,9 \\
\hline LNH T & 9 & 7 & 77,8 \\
\hline Total & 102 & 68 & 66,7 \\
\hline \multicolumn{4}{|l|}{ LH } \\
\hline Escl. Nodular & 13 & 5 & 38,5 \\
\hline Cel. Mixta & 9 & 3 & 33,3 \\
\hline Rico linfocitos & 1 & 1 & 100 \\
\hline Depl. Linf & 1 & 0 & 0 \\
\hline NE & 6 & 0 & 0 \\
\hline Total & 30 & 9 & 30 \\
\hline \multicolumn{4}{|c|}{$\begin{array}{l}\text { EN: Extranodal, LNH: Linfoma no Hodgkin; LH: Linfoma } \\
\text { de Hodgkin. NE: No especificado }\end{array}$} \\
\hline
\end{tabular}

agosto de 2014. Los pacientes enrolados en el estudio firmaron un acta de consentimiento informado al momento de realizar el estudio. Para el análisis de datos se protegió la privacidad y confidencialidad de la información de los pacientes del estudio.

\section{Resultados}

El grupo total de pacientes se muestra en la Tabla 1, correspondió a 102 pacientes con LNH en etapificación (mediana 60,5 años, rango: $15-87$ años, 59,8\% hombres), y a 30 pacientes con LH en etapificación (mediana: 35,5 años, rango: 7-74 años, 53,3\% hombres). Los primeros presentaron significativa mayor edad ( $p<0,0001)$ que el grupo con LH (Figura 1).

\section{Linfoma No Hodgkin}

De los pacientes con LNH ( $n=102)$, un $86,3 \%$ $(88 / 102)$ presentó compromiso ganglionar (Tabla 2 ), siendo las ubicaciones más frecuentes: cervical (61/88: $69,3 \%)$, retroperitoneal $(55,7 \%)$, supraclavicular $(52,3 \%)$, axilar $(48,9 \%)$ y mediastinica $(47,7 \%)$.

El bazo presentó hipercaptación patológica de F18-FDG en 23 pacientes, lo que corresponde al $22,5 \%$ de la muestra.

Un 66,7\% (68/102) tuvo compromiso extranodal al PET/CT, correspondiendo principalmente a médula ósea (29/68: 42,6\%), muscular $(22,1 \%)$, renal $(16,2 \%)$, piel y celular subcutáneo $(16,2 \%)$, intestino $(14,7 \%)$ (Tabla 3). El LNH presentó mayor frecuencia de compromiso extranodal que el LH $(p=0,0007)$.

De los pacientes con compromiso extranodal, $20,6 \%(14 / 68)$ no tenían compromiso ganglionar, es decir presentaron enfermedad extranodal exclusiva. Estos correspondieron principalmente a pacientes con LNH de alto grado o linfoma cutáneo, con lesiones en medula ósea, estómago o piel.

Un 16,7\% (17/102) presentó una tumoración bulky $(\geq 10 \mathrm{~cm}$ ), de ubicación más frecuente extranodal (5 casos), mesentérica (4 casos) y mediastínica (3 casos).

El SUVmax promedio en LNH fue de 13,0 09,7 y varió considerablemente de acuerdo al subtipo histo-

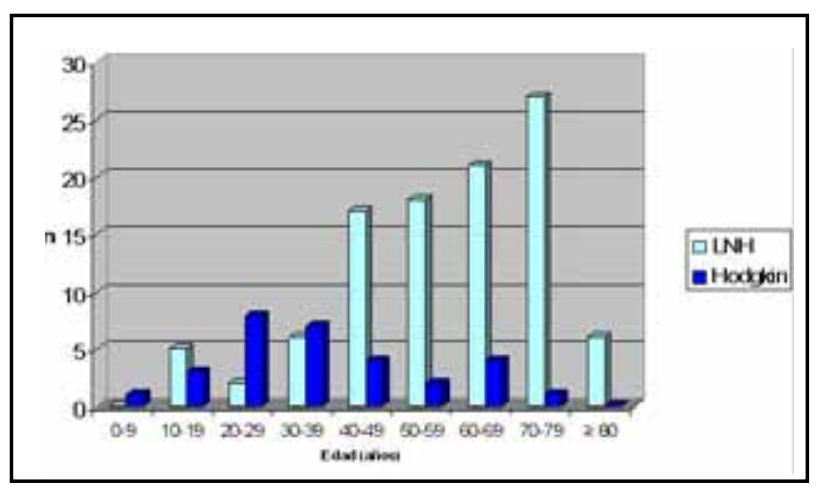

Figura 1. Distribución de frecuencia de pacientes con linfoma según grupo etario. 
Tabla 2. Etapa Ann Arbor, compromiso nodal/extranodal y presencia de Bulky en pacientes con linfoma.

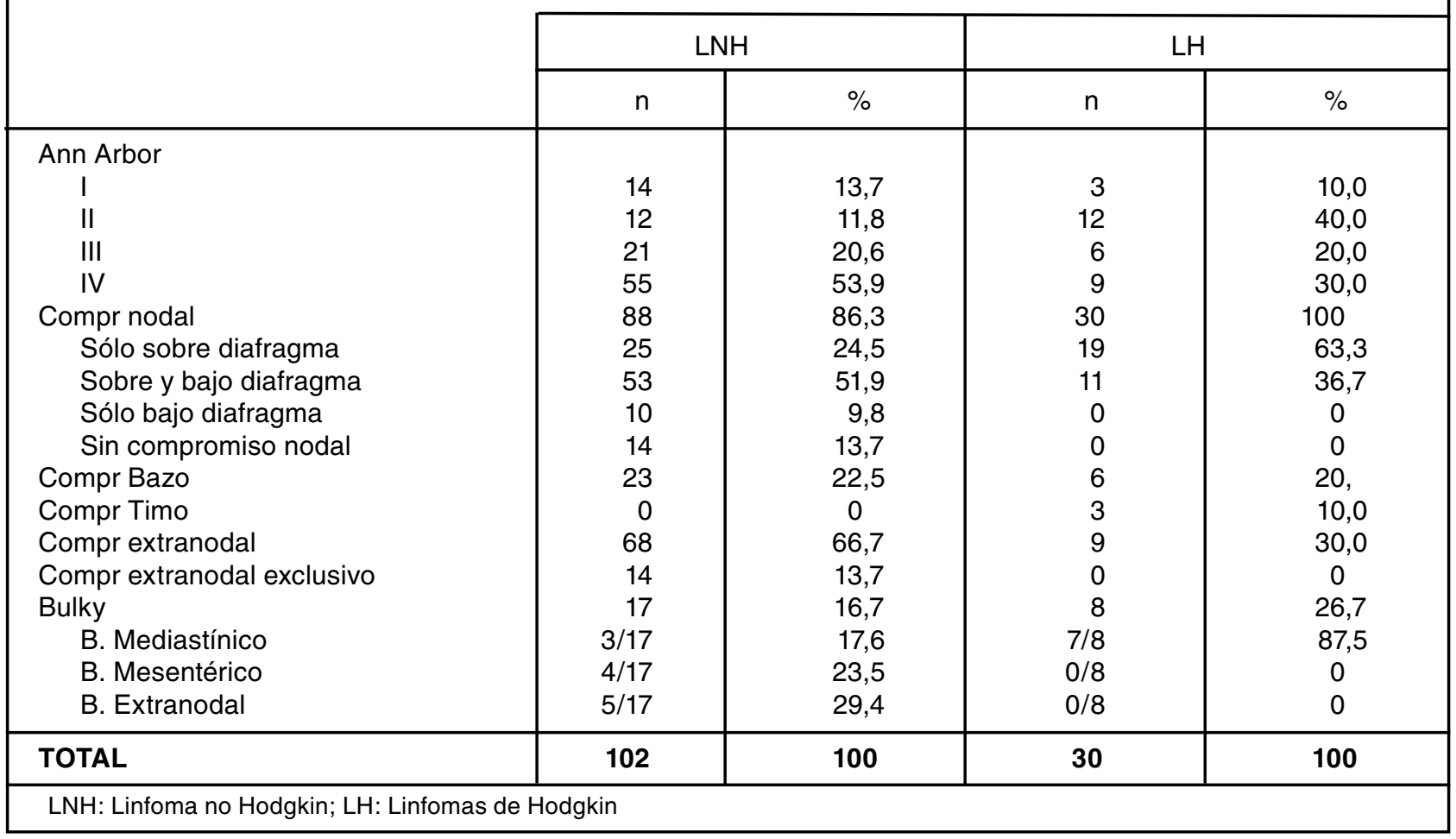

Tabla 3. Distribución del compromiso extranodal en LNH y LH.

\begin{tabular}{|l|c|c|c|c|}
\cline { 2 - 4 } & \multicolumn{2}{c|}{ LNH } & $\mathbf{3}$ & LH \\
\cline { 2 - 5 } & $\mathbf{n}$ & $\%$ & 6 & 66,7 \\
\hline Medula ósea & 29 & 42,6 & 1 & 11,1 \\
Muscular & 15 & 22,1 & 0 & 0 \\
Piel/Celular subcutáneo & 11 & 16,2 & 0 & 0 \\
Renal & 11 & 16,2 & 0 & 0 \\
Intestino & 10 & 14,7 & 0 & 0 \\
Pleura & 8 & 11,8 & 2 & 22,2 \\
Pulmón & 7 & 10,3 & 0 & 0 \\
Estómago & 7 & 10,3 & 1 & 11,1 \\
Peritoneo & 7 & 10,3 & 1 & 1,1 \\
Hígado & 5 & 7,4 & 0 & 0 \\
Glándulas suprarrenales & 5 & 7,4 & 1 & 11,1 \\
Cavidades paranasales & 5 & 7,4 & $\mathbf{9}$ & $\mathbf{1 0 0}$ \\
Total & $\mathbf{6 8}$ & $\mathbf{1 0 0}$ & $\mathbf{9}$ & \\
\hline
\end{tabular}

lógico, como se muestra en Figura 2. Los valores más altos de SUVmax se observaron en Doble Hit (promedio: 20,6, DS: 14,6), y Difuso CG (promedio: 19,9, DS: 10,7), significativamente más altos que cada uno de los otros subgrupos de $\mathrm{LNH}$ tipo $\mathrm{B}(\mathrm{p}<0,05)$. Uno de estos casos se muestra en la Figura 3. Los subtipos de LNH tipo B de menor captación correspondieron a folicular, manto, linfocítico, MALT y tranformación.

EI LNH tipo T obtuvo valores intermedios de SUVmax (promedio: 12,7, DS: 6,8), significativamente mayores que el grupo de LNH de menor captación (promedio: $7,9, \mathrm{DS}: 5,1)$.
El SUVmax mostró correlación con el Ki67 en los casos en que los pacientes contaron con dicho índice proliferativo $(n=39)$, con un $r: 0,623$ y $p<0,0001$.

\section{Linfoma de Hodgkin}

Todos los pacientes con $\mathrm{LH}(\mathrm{n}=30)$ tuvieron compromiso nodal. Los ganglios más frecuentemente afectados fueron los cervicales (27/30: 90\%), supraclaviculares $(80 \%)$, mediastínicos $(76,7 \%)$, hilios pulmonares $(43,3 \%)$, axilares (36,7\%). Los pacientes con LH mostraron significativa menor frecuencia de compromiso ganglionar infradiafragmático que aquellos con LNH $(p<0,0001)$. 

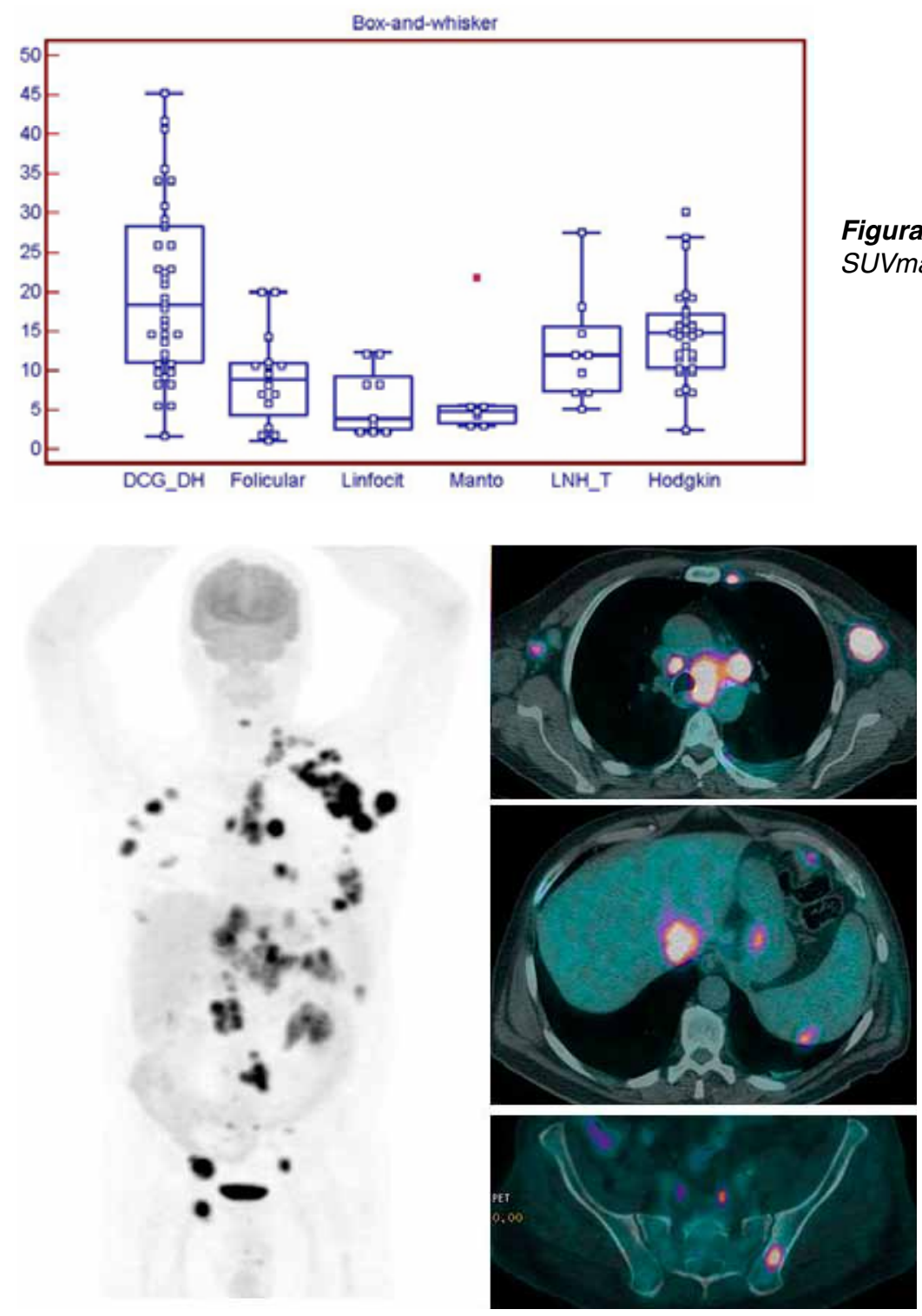

Figura 2. Diagrama "Box and Whisker" de niveles de SUVmax según tipo de Linfoma.
Figura 3. Paciente masculino de 59 años con $L N H$ difuso de células grandes con compromiso nodal a ambos lados del diafragma, y extranodal en médula ósea, bazo, hígado, estómago, riñón izquierdo (no mostrado). SUVmax: 29,2.
El bazo presentó hipercaptación patológica en 6 pacientes, lo que corresponde al $20 \%$ de la muestra. El Timo apareció afectado en 3 pacientes (10\%).

Un 30\% (9/30) tenía compromiso extranodal visible con PET/CT, principalmente de médula ósea (6/9:66,7\%) y pulmón $(22,2 \%)$.

Todos los pacientes con compromiso extranodal presentaron enfermedad ganglionar concomitante.

Ocho pacientes debutaron con Bulky $(26,7 \%)$, siete de ellos de ubicación mediastínica y uno de ubicación cervical. Uno de estos casos se muestra en la Figura 4.

EI LH tuvo valores de SUVmax similar en todos sus subgrupos histológicos, con SUVmax promedio 14,6 (DS:6,0).

\section{Discusión}

El linfoma es una enfermedad linfoproliferativa que afecta primariamente los ganglios y tejido linfoide, y que se clasifica en dos grandes grupos: $\mathrm{LH}$ y LNH.
Ambas son enfermedades diferentes en su tratamiento y pronóstico, aunque su forma de presentación puede ser similar en varios aspectos. El diagnóstico final siempre es de resorte histopatológico y fuertemente dependiente de la evaluación inmunohistoquímica.

Nuestro estudio utilizando PET/CT con F18FDG en una población con linfoma recientemente diagnosticado, no expuesta a tratamiento, aporta una idea general de la extensión y distribución de la enfermedad en una población representativa, y esboza ciertas características propias de algunos tipos y subtipos de linfoma.

El LH correspondería en nuestra muestra a una patología de individuos de entre 20 y 50 años, con enfermedad predominantemente sobre el diafragma, siempre de carácter nodal y con bajo compromiso extranodal. La captación de F18-FDG medida mediante SUVmax es alta en forma invariable en todos los subgrupos histológicos. 


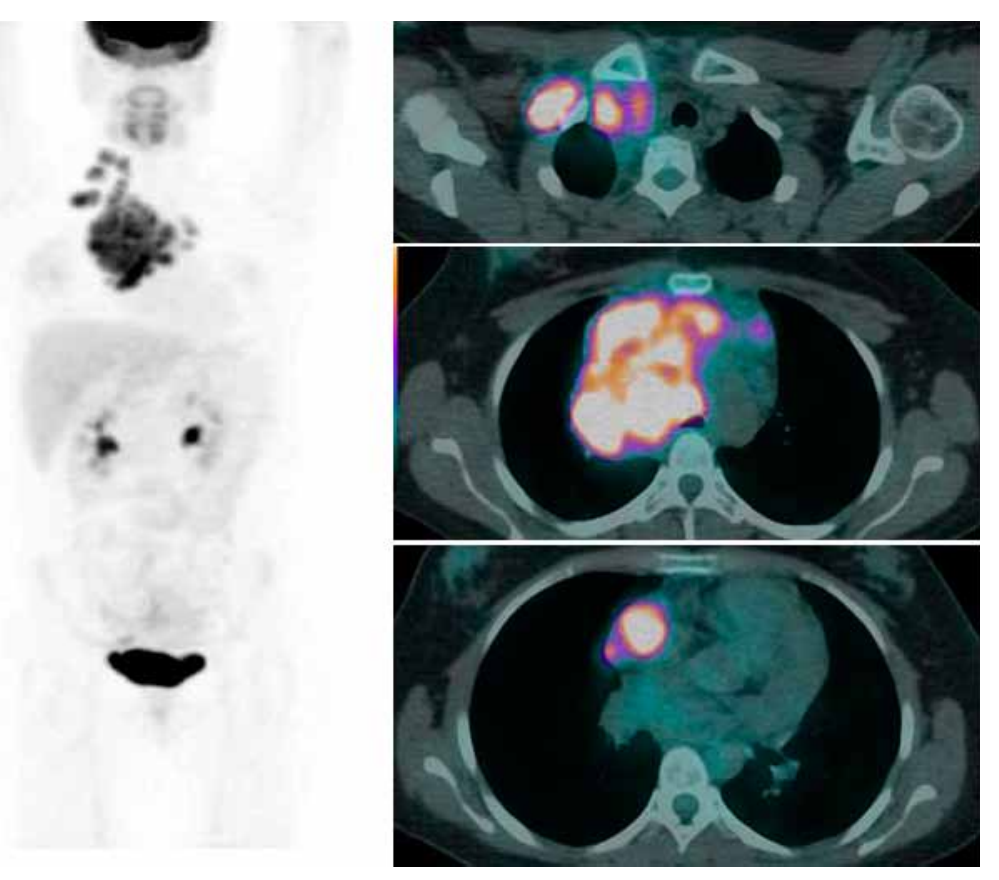

Figura 4. Paciente femenino de 31 años con Linfoma de Hodgkin que presenta compromiso ganglionar cervical, supraclavicular, retropectoral y mediastinico, y bulky mediastínico de alta captación (SUVmax: 19,2).
EI LNH tendría una incidencia creciente con la edad, partiendo desde los 10 años. Presenta enfermedad nodal tanto por sobre como bajo el diafragma, alto compromiso extranodal, y una proporción significativa de enfermedad extranodal exclusiva, en un $14 \%$ de la muestra.

Los valores del SUVmax presentaron alta variabilidad en el $\mathrm{LNH}$, dependiendo mayormente del subtipo histológico y del Ki67, pudiéndose identificar tres grupos de LNH según su avidez de F18-FDG. El de mayor actividad (LNH difuso CG y Doble Hit), el de actividad intermedia ( $L N H$ tipo T), y el de menor actividad (Folicular, Manto, Linfocítico, MALT).

Anderson et al. ${ }^{4}$ publicaron el año 1982 un completo estudio de 473 pacientes con linfoma recientemente diagnosticado, utilizando para ello una exhaustiva evaluación clínica, exámenes de laboratorio de rutina, imágenes radiológicas simples (radiografía de tórax, pielografía endovenosa), imágenes con radioisótopos (cintigrama óseo, exploración sistémica con Galio-67, cintigrafía hepática y esplénica), y técnicas invasivas (aspiración y biopsia de medula ósea, biopsia hepática, laparotomía exploradora). Ellos describen un $67 \%$ de compromiso nodal y un $29 \%$ de compromiso extranodal, este último principalmente de piel/ celular subcutáneo, tracto gastrointestinal, y óseo/ médula ósea.

En una base de datos de 91.306 pacientes con $\mathrm{LNH}$, Glass et al encuentran un $28 \%$ de compromiso extranodal primario, más frecuente de observar en linfomas de alto grado ${ }^{13}$.

El año 2002, Gurney et al. ${ }^{14}$ reportaron un 31,4\% de compromiso extranodal en una serie de 11.334 pacientes con $\mathrm{LNH}$, que afectó principalmente piel
(21,6\%), estómago (13,0\%), intestino delgado (8,2\%), cavidad oral $(7,9 \%)$, tejidos blandos $(6,0 \%)$, glándula tiroidea $(4,8 \%)$. Todos los sitios extranodales fueron confirmados por biopsia e histopatología. Ellos no mencionan los estudios diagnósticos de laboratorio e imágenes realizados a sus pacientes.

El compromiso extranodal fue de alta frecuencia en nuestra casuística, de $30 \%$ en $\mathrm{LH}$ y $67 \%$ en $\mathrm{LNH}$, mayor a lo reportado en las series con etapificación convencional ${ }^{4}$. La enfermedad extranodal fue detectada en mayor o menor medida en todos los subtipos de $\mathrm{LNH}$, con alta frecuencia en Difuso células grandes, Doble HIT, LNH tipo T, MALT, Transformación, frecuencia media en Folicular, y baja frecuencia en Linfocítico y Manto.

El compromiso extranodal afectó principalmente médula ósea y músculo en nuestra serie. Ambas localizaciones son visibles con las imágenes de PET, pero no con las de TC no contrastada ${ }^{7,15}$. Por otro lado, el compromiso renal es tercero en frecuencia, y es fácilmente detectable con TC contrastada, aunque en las imágenes de PET puede ser más dificultoso de definir debido a la excreción fisiológica de F18FDG por la orina ${ }^{16}$. Debido a la complementariedad de ambos estudios, y la posibilidad de realizarlos al mismo tiempo en el equipo PET/CT, creemos que es recomendable utilizar $\mathrm{PET} / \mathrm{CT}$ asociado a TC contrastada en la evaluación inicial del linfoma ${ }^{6,7}$.

Aparte del valor diagnóstico, el PET/CT proporcionaría una estratificación pronóstica en linfomas indolentes de acuerdo al grado de avidez de las lesiones, con pronóstico más pobre en aquellos casos con captación más intensa. En LNH del manto una captación alta se asocia a presencia de blastoides y 
variante de células grandes, lo que se sabe determina un curso más agresivo de la enfermedad ${ }^{15}$. Karma y cols describen que un SUVmax $>5$ se asoció a menor sobrevida en pacientes con LNH del manto (sobrevida a 5 años $34 \%$ vs $87 \%$ en pacientes con SUVmax $\leq 5)^{17}$.

Entonces, en algunos casos la valoración pronóstica pretratamiento dependería no sólo de la extensión de la enfermedad, sino también de la avidez de captación de F18-FDG. Otros índices que estiman de manera precisa la carga tumoral como el volumen tumoral metabólico total (VTM) y glicolisis lesional total (GLT), también han demostrado valor pronóstico en poblaciones determinadas ${ }^{18,19}$. La utilidad de estos índices debe establecerse comparando con parámetros pronósticos conocidos como el Índice Internacional Pronóstico ${ }^{20}$, materia que queda propuesta para un futuro estudio de seguimiento de nuestra casuística.

Cabe mencionar que el PET/CT interino, es decir, el que se realiza después de comenzado el tratamiento, comúnmente entre el $2^{\circ}$ y $3^{\circ}$ ciclo de quimioterapia, representa actualmente la herramienta pronóstica más relevante proporcionada por el PET/ $\mathrm{CT}$, con capacidad de predecir respuesta patológica una vez finalizada la quimioterapia, utilizando para ello criterios cualitativos y semicuantitativos de medición como la escala Deauville de 5 puntos $^{6,8}$.

\section{Conclusiones}

EI PET/CT es el método de elección en la etapificación de pacientes con linfoma, permitiendo detectar enfermedad nodal y extranodal con alta sensibilidad, tanto en LH como en LNH.

\section{Referencias}

1. Swerdlow SH., Campo E., Harris NL. WHO classification of Tumours of Haematopoietic and Lymphoid Tissues. Lyon: IARC; 2008.

2. Freedman A., Caron J., Mauch P., Aster J. Non Hodgkin`s Lymphoma. En: DeVita V., Lawrence T., Rosenberg S., Ed. Lymphomas and Leukemias. China: Wolters Kluwer; 2016, 10th ed: 495-529.

3. Mauch PM., Kalish LA., Kadin M., Coleman CN., Osteen R., Hellman S. Patterns of presentation of Hodgkin disease. Implications for etiology and pathogenesis. Cancer 1993; 71: 2062-2071.

4. Anderson T., Chabner BA., Young RC., Berard CW., Garvin AJ., Simon RM et al. Malignant lymphoma. The histology and staging of 473 patients at the National Cancer Institute. Cancer 1982; 50: 2699-2707.

5. Schaefer NG., Hany TF., Taverna C., Seifert B., Stumpe KDM., von Schulthess GK., et al. Non-Hodgkin lymphoma and Hodgkin disease: coregistered FDG PET and $\mathrm{CT}$ at staging and restaging-do we need contrastenhanced CT? Radiology 2004; 232: 823-829.

6. Cheson BD. Staging and response assessment in lymphomas: The new Lugano classification. Chin Clin Oncol 2015; 4: 1-9.

7. han AB., Barrington SF., Mikhaeel NG., Hunt AA., Cameron L., Morris T., et al. PET-CT staging of DLBCL accurately identifies and provides new insight into the clinical significance of bone marrow involvement. Blood 2013; 122: 61-67.

8. Cheson BD., Fisher RI., Barrington SF., Cavalli F., Schwartz LH., Lister TA., et al. Recommendations for initial evaluation, staging, and response assessment of Hodgkin and non-Hodgkin lymphoma: The Lugano classification. J Clin Oncol 2014; 32: 3059-3069.

9. Jaffe ES., Harris NL., Stein H. WHO Classification of Tumours of Haematopoietic and Lymphoid Tissues. Lyon, France: IARC Press; 2001.

10. Lin EC., Alavi A., Kinahan P. Standarized uptake value. En: Lin EC, Alavi A. PET and PET/CT: A clinical guide. New York, USA: Thieme Medical Publishers Inc; 2005: 28-31.

11. Gurney KA., Cartwright RA. Increasing incidence and descriptive epidemiology of extranodal non-Hodgkin lymphoma in parts of England and Wales. Hematology J 2002; 3: 95-104.

12. National Comprehensive Cancer Network. NCCN Guidelines. Disponible en: http://www.nccn.org/ professionals/physician_gls/f_guidelines.asp\#site. [Consultado el 10 de julio de 2017].

13. Glass AG., Karnell LH., Menck HR. The National Cancer Data Base report on non Hodgkin's lymphoma. Cancer 1997; 80: 2311-2320.

14. Gurney KA., Cartwright RA. Increasing incidence and descriptive epidemiology of extranodal non-Hodgkin lymphoma in parts of England and Wales. Hematology Journal 2002; 3: 95-104.

15. Gallamini A., Borra A. Role of PET in Lymphoma. Curr Treat Options Oncol 2014; 15: 248-261.

16. Blake MA., Singh A., Setty BN., Slattery J., Kalra M., Maher MM., et al. Pearls and Pitfalls in Interpretation of Abdominal and Pelvic PET-CT. RadioGraphics 2006; 26: 1335-1353.

17. Karam M., Ata A., Irish K., Feustel PJ., Mottaghy FM., Stroobants SG., et al. FDG positron emission tomography/computed tomography scan may identify mantle cell lymphoma patients with unusually favourable outcome. Nucl Med Commun 2009; 30: 770-778.

18. Ceriani L., Martelli M., Zinzani PL., Ferreri AJM., Barbara Botto B., Stelitano C., et al. Utility of baseline 18FDG-PET/CT functional parameters in defining prognosis of primary mediastinal (thymic) large B-cell lymphoma. Blood 2015; 126: 950-956.

19. Kanoun S., Rossi C., Berriolo-Riedinger A., Dygai-Cochet I., Cochet A., Humbert O., et al. Baseline metabolic tumour volume is an independent prognostic factor in Hodgkin lymphoma. Eur J Nucl Med Mol Imaging 2014; 41: 1735-1743.

20. Ziepert M., Hasenclever D., Kuhnt E., Glass B., Schmitz N., Pfreundschuh M., et al. Standard International Pronostic Index remains a valid predictor of outcome for patients with aggressive CD 20+ B-cell Lymphoma in the rituximab era. J Clin Oncol 2010; 28: 2373-2380. 